\title{
Circulating malondialdehyde modified $L D L$ is a biochemical risk marker for coronary artery disease
}

\author{
T Amaki, T Suzuki, F Nakamura, D Hayashi, Y Imai, H Morita, K Fukino, T Nojiri, S Kitano, N Hibi, \\ T Yamazaki, R Nagai
}

$\mathrm{O}$ xidatively modified low density lipoprotein (OxLDL) plays an important role in the development of atherosclerosis as its uptake by macrophages and smooth muscle cells leads to formation of foam cells which is a critical step in the evolution of the pathological state. ${ }^{12}$ Circulating OxLDL concentrations may therefore reflect the state of pathological atherosclerosis, and be a possible biochemical risk marker for coronary artery disease (CAD). Numerous efforts have been directed at detecting OxLDL concentrations in the circulation for this reason, but technical difficulties have hampered detection of minute amounts of OxLDL. To overcome these limitations, we focused on circulating malondialdehyde modified LDL (MDA-LDL), a chemical modification thought to reflect naturally occurring oxidation of LDL, ${ }^{3}$ and developed a sensitive immunoassay of circulating MDA-LDL concentrations. The diagnostic performance of MDA-LDL in CAD was compared against known lipid markers. This comparison revealed, for the first time, that MDA-LDL is superior, thus suggesting that MDALDL may be a promising tool for the biochemical detection of CAD.

\section{METHODS}

Consenting patients with CAD defined as having greater than $75 \%$ stenosis in one or more arteries on coronary angiography were enrolled, as were normal control subjects which included patients with normal coronary angiograms, and subjects who were admitted for regular health examinations and had: (1) no history of CAD; (2) normal renal function; (3) normal ECG and chest $x$ ray.

Blood was drawn under fasting conditions and centrifuged within four hours. Stabilising reagent containing sucrose and EDTA was added and samples were stored at $-20^{\circ} \mathrm{C}$ until the time of assay, which was within 28 days. MDA-LDL concentrations were measured by a sandwich enzyme linked immunosorbent assay (ELISA) procedure using an antiMDA-LDL monoclonal antibody as the capture antibody and an anti-human apolipoprotein B monoclonal antibody labelled by $\beta$ galactosidase as previously described with slight modifications. ${ }^{5}$ The assay specifications were as follows: the measuring range of the assay was from 12.5$400 \mathrm{U} / \mathrm{l}$; within run reproducibility, as a measure of analytical precision, showed a coefficient of variance of $5.6 \%$; recovery, as a measure of analytical accuracy and defined as the observed versus expected value when concentrated MDA-LDL was added to patient serum, was $98 \%$. One unit per litre of MDA-LDL was defined as the absorbance obtained with the standard at a concentration of $1 \mathrm{mg} / \mathrm{l}$. Circulating concentrations of total cholesterol (normal reference 150-219 mg/dl), LDL cholesterol (70-139 mg/dl), high density lipoprotein (HDL) cholesterol (4l-96 mg/dl), triglyceride (50-149 mg/ $\mathrm{dl})$, and apoprotein B (66-109 mg/dl) were also measured.
Statistical analysis was done using the unpaired $t$ test for analysis of two groups and the Kruskal-Wallis test for effects of age. Data are shown as mean (SD) and a probability value of $\mathrm{p}<0.05$ was considered significant.

\section{RESULTS}

Fifty three patients with CAD ( 43 males and 10 females, aged 65.3 (9.4) years) and 57 normal controls (46 males and 11 females, aged 50.4 (13.1) years) were enrolled. Comparison of baseline characteristics (fig 1A) showed similar total cholesterol, LDL, triglyceride and apoprotein B concentrations between groups, but higher age and lower HDL cholesterol concentrations in CAD patients. MDA-LDL concentrations were notably raised in CAD patients (CAD 104.8 (42.9) U/l $v$ control subjects $76.0 \quad(23.3) \mathrm{U} / \mathrm{l}, \mathrm{p}<0.0001)$. Lack of association of MDA-LDL concentrations with age either for patients or controls ruled out age dependent effects (data not shown). Higher MDA-LDL concentrations were seen in severe CAD as manifested by the greater number of diseased vessels ( single vessel disease (SVD) 102.6 (39.5) U/l, $\mathrm{n}=21$; two vessel disease (2VD) 95.6 (38.8) U/l, $\mathrm{n}=24$; three vessel disease (3VD) 138.1 (52.0) $\mathrm{U} / \mathrm{l}, \mathrm{n}=8 ; \mathrm{p}=0.02$ for SVD $v$ $3 \mathrm{VD}$ and for $2 \mathrm{VD} v 3 \mathrm{VD})$. Analysis according to degree of stenosis showed a tendency for total occlusion lesions to show slightly higher concentrations although not significantly higher ( $\leqslant 75 \%$ stenosis, $103.4(46.3) \mathrm{U} / \mathrm{l}, \mathrm{n}=10$; $\leqslant 90 \%, 85.0(27.0) \mathrm{U} / \mathrm{l}, \mathrm{n}=9 ; \leqslant 99 \%, 89.2$ (18.8) U/l, $\mathrm{n}=4 ; 100 \%, 120.1 \quad(56.7) \mathrm{U} / \mathrm{l}, \mathrm{n}=17)$. Of the CAD patients, nine patients had acute coronary syndromes (ACS) such as acute myocardial infarction and unstable angina, and all remaining patients had stable CAD (that is stable angina, post-intervention re-study, post-bypass angiogram). There was no significant difference in MDA-LDL concentrations between ACS and stable CAD patients suggesting that unstable plaque pathology does not affect concentrations. Of 27 patients with hyperlipidaemia, 22 received statins for more than three months; this did not affect the findings. MDA-LDL concentrations were elevated in CAD patients not receiving statins (CAD patients without statins 101.5 (42.7) U/l, $\mathrm{n}=31$; control 76.0 (23.3) U/l, $\mathrm{n}=57, \mathrm{p}=0.0005)$. There was also no difference in MDALDL concentrations between the 22 patients receiving statins from the other CAD patients (patients receiving statins 109.3 (43.8) U/l, n = 22; other CAD patients 101.5 (42.7) U/l, $\mathrm{n}=31, \mathrm{p}=0.52)$. Furthermore, MDA-LDL concentrations in patients with CAD did not differ from control patients regardless of history of diabetes mellitus, hypertension or smoking habit.

Abbreviations: $A C S$, acute coronary syndromes; $C A D$, coronary artery disease; HDL, high density lipoprotein; LDL, low density lipoprotein; MDA, malondialdehyde-modified; Ox, oxidative; SVD, single vessel disease; 2VD, two vessel disease; 3VD, three vessel disease. 
A

\begin{tabular}{llll}
\hline & CAD & Controls & $\mathrm{P}$ \\
\hline $\mathrm{n}$ & 53 & 57 & \\
Age, y & $65.3(9.4)$ & $50.4(13.1)$ & $<0.001$ \\
Men, $\mathrm{n}(\%)$ & $43(81 \%)$ & $46(81 \%)$ & 0.954 \\
Hypertension, \% & 81.1 & 24.6 & $<0.001$ \\
Diabetes mellitus, \% & 34 & 1.8 & $<0.001$ \\
Smoking, \% & 60.4 & 36.8 & 0.014 \\
MDA-LDL (U/l) & $104.8(42.9)$ & $76.0(23.3)$ & $<0.0001$ \\
Total cholesterol (mg/dl) & $186.3(29.5)$ & $198.1(38.2)$ & 0.0738 \\
LDL (mg/dl) & $115.3(23.2)$ & $121.6(33.6)$ & 0.2614 \\
HDL (mg/dl) & $46.6(12.2)$ & $54.3(14.9)$ & 0.0039 \\
Triglyceride (mg/dl) & $108.2(56.0)$ & $123.5(71.8)$ & 0.2213 \\
apoB (mg/dl) & $95.9(20.9)$ & $97.6(26.9)$ & 0.715 \\
\hline
\end{tabular}

B

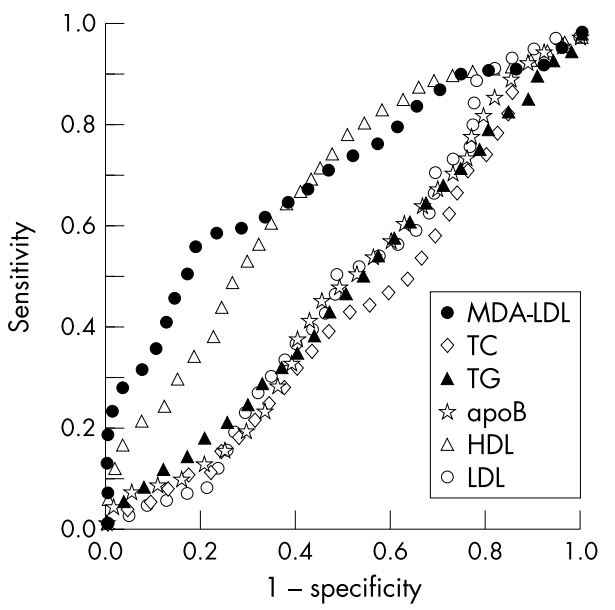

C

\begin{tabular}{llllll}
\hline Item & Cut-off & Sensitivity & Specificity & PPV & NPV \\
\hline MDA-LDL & $85.6 \mathrm{U} / \mathrm{l}$ & 0.64 & 0.65 & 0.63 & 0.66 \\
Total cholesterol & $193 \mathrm{mg} / \mathrm{dl}$ & 0.43 & 0.49 & 0.44 & 0.48 \\
LDL & $113 \mathrm{mg} / \mathrm{dl}$ & 0.47 & 0.47 & 0.45 & 0.49 \\
HDL & $49 \mathrm{mg} / \mathrm{dl}$ & 0.68 & 0.60 & 0.61 & 0.67 \\
Triglyceride & $102 \mathrm{mg} / \mathrm{dl}$ & 0.47 & 0.49 & 0.46 & 0.50 \\
apoB & $97 \mathrm{mg} / \mathrm{dl}$ & 0.45 & 0.53 & 0.47 & 0.51
\end{tabular}

$\mathrm{PPV}=$ positive predictive value, $\mathrm{NPV}=$ negative predictive value

Diagnostic implications of MDA-LDL concentrations against other lipid markers were assessed by receiver operating characteristics curve analysis. The analysis demonstrated MDA-LDL to show superior performance against the other parameters in our study population (fig 1B). MDA-LDL concentrations at a cut-off level of $85.6 \mathrm{U} / \mathrm{l}$ showed a sensitivity and specificity of $64 \%$ and $65 \%$, respectively (area under the curve 0.72). Odds ratio showed a 3.3-fold likelihood for patients with raised MDA-LDL concentrations to have coronary artery disease. The other lipid parameters showed the following diagnostic performance (as shown in order of highest area under the curve): HDL cholesterol (0.67), apoprotein B (0.49), LDL cholesterol $(0.45)$, triglyeride (0.45), and total cholesterol (0.40). Sensitivity, specificity as well as positive and negative predictive values are shown (fig 1C). Therefore, MDA-LDL was the most superior lipid marker of those tested in this study.

\section{DISCUSSION}

Our study shows increased serum concentrations of MDALDL in patients with CAD. MDA-LDL is an independent risk
Figure 1 (A) Baseline characteristics of participants. (B) Receiver operating characteristics (ROC) curve analysis of MDA-LDL and other lipid markers in patients with CAD. (C) Sensitivity, specificity, and positive and negative predictive values are shown. factor of CAD as there was no association with other risk factors such as hypertension, hyperlipidaemia, smoking habit, or sex. MDA-LDL concentrations are higher in patients with severe disease, such as multi-vessel disease, which shows that not only are MDA-LDL concentrations raised in patients with CAD but also that the concentrations reflect the severity of the pathogenic state. Receiver operating characteristic curve analysis showed superior performance of association between MDA-LDL and CAD as compared to other lipid markers, which is the first comparison to our knowledge. The conclusions of our study are limited given the small patient study population, but suggest that MDALDL is a promising lipid parameter to assess the risk of CAD.

Limitations of the present study include: (1) biased populations which analysed very sick patients versus very healthy patients; (2) the new measure was performed in a single centre laboratory; (3) the blood sampling protocol was ideal which may overestimate the clinical utility.

Future questions which remain to be answered are the prognostic and therapeutic roles of MDA-LDL concentrations in CAD. Will patients with higher concentrations of 
MDA-LDL be prone to CAD in later life? Will MDA-LDL concentrations be a therapeutic parameter to assess risk for CAD (for example, lipid lowering)? These questions will need to be clarified in future studies before MDA-LDL concentration can become established as a powerful diagnostic lipid parameter of CAD.

\section{ACKNOWLEDGEMENTS}

The authors thank Dr Takayuki Shindo, Hiroshi Nishimura, Koshiro Monzen and all members of the cardiac catheterisation lab at the University of Tokyo for providing samples.

\section{Authors' affiliations}

T Amaki, T Suzuki, F Nakamura, D Hayashi, Y Imai, H Morita, K Fukino, T Nojiri, T Yamazaki, R Nagai, Department of Cardiovascular Medicine, The University of Tokyo, Tokyo, Japan

S Kitano, N Hibi, SRL Inc, Tokyo, Japan
Correspondence to: Dr T Suzuki, Department of Cardiovascular Medicine, University of Tokyo, 7-3-1 Hongo, Bunkyo-ku, Tokyo 1138655, Japan; torusuzu-tky@umin.ac.jp

\section{Accepted 19 January 2004}

\section{REFERENCES}

1 Steinberg D, Parthasarathy S, Carew TE, et al. Beyond cholesterol. Modifications of low-density lipoprotein that increase its atherogenicity. N Engl J Med 1989;320:915-24.

2 Ross R. The pathogenesis of atherosclerosis: a perspective for the 1990s Nature 1993;362:801-9.

3 Haberland ME, Fong D, Cheng L. Malondialdehyde-altered protein occurs in atheroma of Watanabe heritable hyperlipidemic rabbits. Science 1988;241:215-8.

4 Fogelman AM, Shechter I, Seager J, et al. Malondialdehyde alteration of low density lipoproteins leads to cholesteryl ester accumulation in human monocyte-macrophages. Proc Natl Acad Sci U S A 1980;77:2214-8.

5 Kotani K, Maekawa M, Kanno T, et al. Distribution of immunoreactive malondialdehyde-modified low-density lipoprotein in human serum. Biochim Biophys Acta 1994;1215:121-5.

\section{IMAGES IN CARDIOLOGY}

\section{Magnetic resonance angiography of pseudocoarctation}

$\Delta$ 40 year old woman presented with an abnormal chest radiograph without any symptoms. She had no history of hypertension. On examination she had a regular pulse and normal range of blood pressure measured at each extremity. The chest radiograph revealed a large mass with features suggesting an aortic anomaly including peripheral linear calcification and a tubular and tortuous opacity, the outline of which was smoothly continuous to the descending thoracic aorta (panel A). Conventional $x$ ray aortography failed because the catheter inserted via the right femoral artery could not pass over the tortuous aortic arch in order to get the tip located proximal to the arch for contrast injection. The patient underwent contrast enhanced, magnetic resonance angiography. Three dimensional reconstructed images demonstrated an extremely elongated and approximately three-turned, helical, aortic arch with multiple saccular aneurysms in the anteroposterior (panel B) and oblique lateral (panel C) views. The more caudal origin of the left subclavian artery was clearly depicted in the posterior view of the three dimensional image (arrow in panel C). This congenital anomaly consisting of redundancy of the aortic arch without any significant obstruction has been known as pseudocoarctation. Surgical intervention was considered but rejected because of the absence of symptoms, complications, any evidence of risk of rupture, and associated cardiac abnormalities related to pseudocoarctation in this patient.
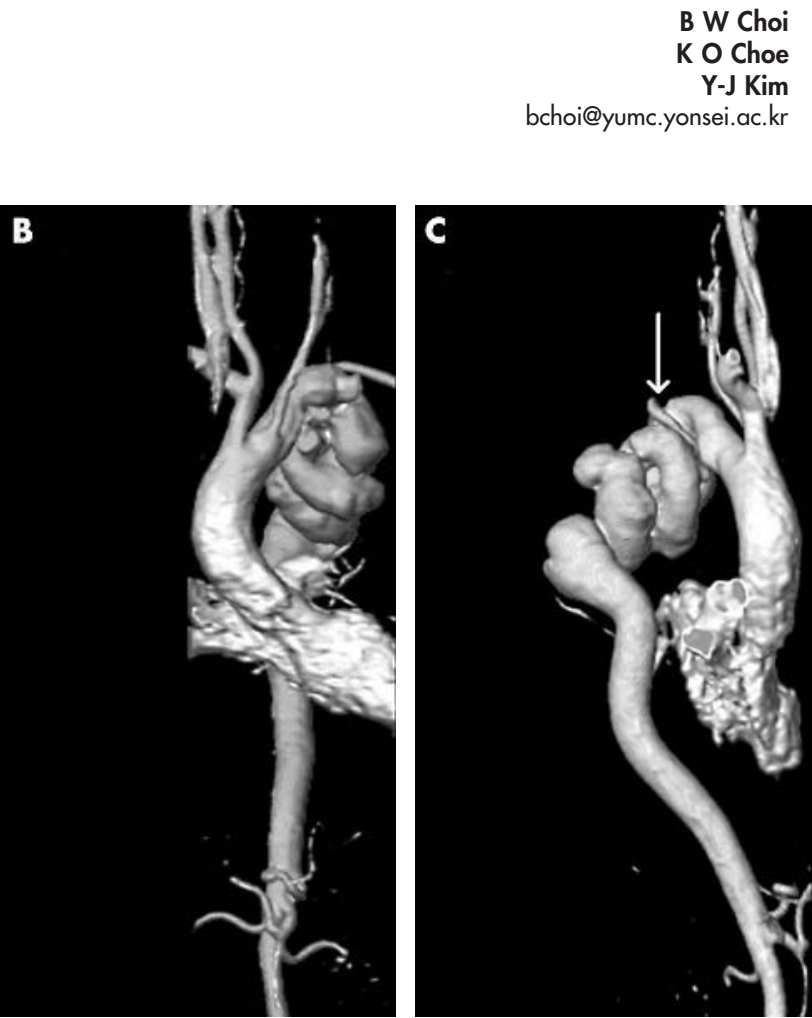